\title{
Editorial
}

doi: http://dx.doi.org/10.14718/revfinanzpolitecon.2014.6.1.1

Joan Miguel Tejedor Estupiñán ${ }^{1}$

\section{La economía regional, algunos contextos e implicaciones}

Antes de presentar a nuestros lectores este número especial dedicado a los temas relacionados con la economía regional, tengo el honor de comunicarles que el pasado 27 de diciembre de 2013 se divulgaron los resultados de la I Actualización de 2013 de la Base Bibliográfica Nacional de PublindexColciencias, en la cual la revista Finanzas y Política Económica fue clasificada en la categoría "B". Este resultado evidencia el compromiso y los esfuerzos realizados por el equipo de la revista conformado por su director, el doctor William Gilberto Delgado Munevar, el editor (quien escribe estas líneas), la Facultad de Ciencias Económicas y Administrativas y su Coordinación de Investigaciones, nuestros colaboradores del comité editorial, científico y grupo de árbitros nacionales e internacionales, y la Unidad Editorial de la Universidad Católica de Colombia, quienes nos hemos trazado el objetivo de garantizar la calidad editorial y científica de su contenido, cumpliendo con los estándares de indización nacionales y extranjeros. Junto a este logro, es necesario reconocer también que, en su recorrido, la revista ha sido incluida en sistemas de indexación a nivel mundial como la Red de Revistas Científicas de América Latina y el Caribe, España y Portugal (Redalyc), el Directory of Open Access Journals (DOAJ), EBSCO, ECONLIT, Clase, Dotec-Colombia, Repec, Dialnet, E-revistas, Actualidad Iberoamericana y Ulrich's Periodical Directory, lo cual también ha impulsado su impacto y visibilidad, y ha permitido que se consolide como fuente académica y de investigación dentro de la amplia literatura de las ciencias económicas; lo anterior también genera espacios para la integración regional desde los ámbitos académicos e investigativos.

Guiados por estos objetivos, en esta edición especial queremos abordar la idea de economía regional, siendo conscientes, desde la perspectiva de Serrano (2008), de que en la actualidad el concepto de región ha trascendido los conceptos que la referenciaban desde la interdependencia de los Estados ligados política, económica y geográficamente (los cuales son aparentemente homogéneos), o a los que la entienden como un subsistema supranacional del sistema internacional, así como otros que la observan como formación regional emergente con una dinámica propia.

Al respecto, la realidad nos muestra cómo los países fronterizos pueden tener los más complejos conflictos, los cuales los alejan de las posibilidades del desarrollo, o cómo países como Colombia tienen dentro de sus principales socios comerciales a la Unión Europea (UE) y Estados Unidos, siendo así evidente que la economía regional no tiene que ver solamente con la integración económica, sino en detenerse en las problemáticas relacionadas con la cohesión social y la cooperación para la paz, la libertad y el desarrollo a nivel local, regional y global, lo cual se traduce en la utopía de alcanzar el respeto por los derechos humanos en el mundo.

1 Magíster en Derechos Humanos y economista. Editor de la Revista Finanzas y Política Económica de la Universidad Católica de Colombia. Dirección de correspondencia: Facultad de Economía, Universidad Católica de Colombia, Carrera 13 N. 47-49 (Bogotá, Colombia). Correo electrónico: jmtejedor@ucatólica.edu.co. 
Este número especial de la revista contiene ocho documentos que abordan este problema anteriormente descrito de manera general. En el primer artículo, Henry Antonio Mendoza Tolosa y Carlos Andrés Yanes Guerra, de la Universidad Católica de Colombia, estudian el impacto que tiene el gasto y la inversión pública en el crecimiento económico de los departamentos en Colombia, basados en las cuentas nacionales para el periodo 2000-2011. Apoyados en un modelo estadístico, estiman la producción departamental por rama de actividad, e identifican la estructura productiva de dichas regiones, y sus cambios a lo largo del tiempo.

Los resultados se obtienen a partir de un modelo de panel de datos de efectos fijos que refleja las diferencias existentes entre los departamentos, y el impacto del gasto y la inversión con la temporalidad e información disponible, lo cual indica que el gasto y la inversión públicos inciden directamente en la dinámica económica departamental; también se evidencian los efectos en las economías grandes más ricas y en las economías departamentales pequeñas y pobres.

En el segundo artículo, los profesores Antonio Rafael Peña Sánchez, Mercedes Jiménez García, José Ruiz Chico, de la Universidad de Cádiz, analizan el impacto de la crisis económica sobre las distintas regiones y los sectores de la economía española desde el año 2007 al 2010; estudian la evolución del mercado de trabajo y el capital humano en los últimos años. Se destaca cómo el descenso experimentado por el empleo, y en menor medida por el capital humano, evidencia la vulnerabilidad del tejido productivo y empresarial de las regiones españolas. Los resultados obtenidos muestran que la crisis económica ha afectado de forma desigual a las regiones españolas, intensificándose principalmente en aquellos territorios con mayor especialización en sectores como la construcción, la industria y los servicios destinados a la venta.

En el tercer artículo, Silvia Bou Ysàs y Magda Cayón Costa, de la Universidad Autónoma de Barcelona, y Albert Hernández, del Banco de Sabadell (Madrid), partiendo del supuesto que, como herramienta para una correcta política de empleo, la legislación laboral debe sentar las bases sólidas para que en el momento de recuperación económica se generen puestos de trabajo estables, presentan un modelo de simulación de contratación que asimila el comportamiento del empresario al del propietario de una cartera de inversión compuesta por dos derivados reales, un swap y una opción de venta americana; este modelo también les permite hacer un análisis de sensibilidad de las diferentes variables que intervienen en la contratación, y, además, analizar los efectos que la última reforma laboral española tiene sobre la decisión de contratar del empresario. Los resultados muestran que la variable que más sensibilidad presenta sobre la contratación es la cuota patronal de la seguridad social, y se concluye que la reducción de los costes de despido no acelera la decisión de contratar.

En el cuarto artículo, Eliana Scialabba y Mariano Carpineti, de la Universidad de Ciencias Empresariales y Sociales, Buenos Aires, muestran que Argentina cuenta con una gran cantidad de recursos naturales, específicamente los ligados al complejo oleaginoso, que se relacionan con los cambios en la demanda mundial y en los procesos de producción, que han permitido la conformación de un complejo productivo en torno a ellos, el cual se encuentra en su etapa madura, que orienta su producción a la exportación, y genera tanto valor como también externalidades positivas, tanto en el empleo como en el desarrollo sustentable. Finalmente, afirman que la producción de biodiesel en 
torno al complejo oleaginoso agrega valor a la producción, y genera encadenamientos hacia atrás, adelante y hacia los lados, lo que origina la creación de actividades complementarias.

En el quinto artículo, Pedro Barrientos Felipa, de la Universidad Nacional Mayor de San Marcos, de Lima, Perú, explica que en el mercado internacional existen países que tienen características similares de oferta, por lo que es conveniente establecer una ventaja sobre los competidores de manera que asegure y fortalezca el crecimiento económico. Describe que los compradores buscan los productos con los mejores atributos, que satisfagan sus necesidades $y$, de esta manera, se hace conveniente el establecimiento de una marca país. De esta manera, explica cómo las naciones realizan una serie de actividades para generar un país (marca) posicionado, y la marca es así consecuencia de diversas actividades nacionales. Identifica la importancia de la marca país en el comercio internacional, y las actividades necesarias para que sea sostenible.

En el sexto artículo, Gabriel Eduardo Escobar Arias, de la Universidad Nacional de Colombia (sede Manizales), relaciona los flujos de efectivo con el entorno económico de la empresa, y destaca la importancia de que las decisiones tanto de inversión, financiación y de operación deben ser resultado del medio en el cual se realizan las actividades comerciales. Así mismo, determina la relación entre los flujos de efectivo de las empresas de Risaralda del sector de servicios y el comportamiento del entorno económico regional en el periodo 2002-2011. El autor muestra los flujos de efectivo de las empresas del sector y las principales variables macro; finalmente, los resultados obtenidos evidencian que no existe una correlación entre los flujos de efectivo y las variables macro.

En el séptimo artículo, Juma'h Ahmad Hussein, de la Universidad Interamericana de Puerto Rico, discute acerca de las implicaciones de la materialidad de la información financiera sobre el "manejo de los ingresos" y sus implicaciones frente a la descripción y el análisis del contenido de la Codificación de Normas Contables ${ }^{\mathrm{TM}}$. Muestra cómo las perspectivas profesionales son utilizadas para confirmar la ausencia de directrices con importancia relativa en los Principios de Contabilidad Generalmente Aceptados (en inglés PCGA) de los EE. UU., devela cómo los términos de materialidad, significancia e importancia han sido usados para determinar la inclusión de la materialidad en las diferentes normas, y llega a la conclusión de que las motivaciones internas y externas influyen en las prácticas de "manejo de ganancias", y que los PCGA de EE. UU. no cuentan con directrices bien definidas para aplicar la materialidad en la toma de decisiones.

Finalmente, en el octavo documento, Rodrigo Pérez Peña, de la Universidad Piloto de Colombia, explica que la ciudad de Girardot es el principal centro turístico de la región del Alto Magdalena donde se desarrolla la industria, el comercio y los servicios, lo que contribuye al desarrollo de la ciudad. Muestra cómo la ciudad y la región adolecen de fuentes para determinar las necesidades de inversión en los diferentes sectores económicos, por lo que los inversionistas o las empresas interesadas en realizar las inversiones se abstengan de hacerlas, y se alejan de la posibilidad de generar empleo y desarrollo. A partir de la consulta a los actores actuales, se elaboraron indicadores, los cuales podrán ser consultados por los inversionistas para que identifiquen los sectores económicos y financieros donde existen las mejores oportunidades de inversión. 
EDITORIAL

\section{REFERENCIAS}

Publindex. (27 de diciembre 2013). Resultados I Actualización del IBN Publindex 2013. Recuperado de http://201.234.78.173:8084/publindex/

Serrano, L. O. (2008). Sobre la naturaleza de la integración regional: teorías y debates. Revista de Ciencia Política, 28(2). Recuperado de http://www.redalyc.org/articulo. oa?id=32414669004 